\title{
Evaluación de las isotermas de sorción de granos y harina de kiwicha (Amaranthus caudatus)
}

\section{Evaluation of sorption isotherms of grains and flour of amaranth (Amaranthus caudatus)}

\section{Avaliação das isotermas de sorção de grãos e farinha de amaranto (Amaranthus caudatus)}

\author{
David Choque-Quispe ${ }^{1 *}$; Carlos A. Ligarda-Samanez ${ }^{1}$; Betsy S. Ramos-Pacheco ${ }^{1}$; Fredy Taipe-Pardo ${ }^{1}$; \\ Diego E. Peralta-Guevara'; Aydeé M. Solano Reynoso ${ }^{2}$ \\ ${ }^{1}$ Universidad Nacional José María Arguedas, C.P. Ingeniería Agroindustrial, Andahuaylas, Apurímac, Perú, \\ 2Universidad Tecnológica de los Andes, Andahuaylas, Apurímac, Perú \\ *davidchoqueq@hotmail.com
}

Fecha recepción: 29 de mayo de 2017 Fecha aceptación: 10 de septiembre de 2018

\begin{abstract}
Resumen
El objetivo principal fue evaluar las isotermas de sorción de granos y harina de kiwicha (Amaranthus caudatus) de las variedades Oscar Blanco, Noel Vietmeyer y CICA, se utilizó el método estático gravimétrico para intervalos de humedad relativa de 10 a $90 \%$ a temperaturas de $18,20,25$ y $30{ }^{\circ} \mathrm{C}$. Se estudiaron 11 modelos matemáticos y se modelaron a datos experimentales a través de RNL tomando como criterio de convergencia el coeficiente de correlación $R^{2}$ y el error medio relativo \%E, así el modelo de Adam y Shove presentó mejor ajuste para los granos de kiwicha, mientras que para la harina fue el modelo de Peleg. Asimismo, se observó que la temperatura de almacenamiento influye significativamente $(p$-value $<0,05)$ en la humedad de equilibrio $\left(X_{e}\right)$ de los granos y harina de kiwicha. El calor isostérico de adsorción oscila entre 6,340 a 0,235 $\mathrm{KJ} / \mathrm{g}$ para humedades de 0,06 a 0,15 $\mathrm{g}$ de agua/g $\mathrm{m}$.s. para los granos de la variedad Oscar Blanco, para la variedad Noel Vietmeyer oscila entre 2,556 a 0,518 $\mathrm{KJ} / \mathrm{g}$ para humedades de 0,04 a $0,12 \mathrm{~g}$ de agua/g m.s., y para el grano CICA de 4,645 a 0,176 $\mathrm{KJ} / \mathrm{g}$ para humedades de 0,06 a 0,14 g de agua/g m.s, en tanto que para la harina de la variedad Oscar Blanco se encuentra entre 32,028 a $1,99 \mathrm{KJ} / \mathrm{g}$ para humedades de 0,05 a $0,12 \mathrm{~g}$ de agua/g m.s., y para la variedad Noel Vietmeyer de 26,992 a 0,648 KJ/g para humedades de 0,06 a 0,20 $\mathrm{g}$ de agua/g m.s.
\end{abstract}

Palabras clave: Kiwicha, adsorción, humedad de equilibrio $\left(X_{e}\right)$, calor isostérico.

\begin{abstract}
The main objective was to evaluate the adsorption isotherms of grain and flour amaranth (Amaranthus caudatus) varieties Oscar White, Noel Vietmeyer and CICA, it was used gravimetric method for static intervals relative humidity 10 to $90 \%$ at temperatures of were studied 11 mathematical models and they were modeled to experimental data through RNL, taking as a convergence criterion the correlation coefficient $R^{2}$ and the average relative error \%E, thus the model of Adam and Shove present best fit for grains of amaranth, while the flour was Peleg model. Was also observed that the storage temperature influences significantly $(p$-value $<0.05)$ in the equilibrium moisture $(X e)$ grain and flour amaranth. The heat isosteric of adsorption oscillates between 6.340 to $0.235 \mathrm{~kJ} / \mathrm{g}$ for moisture content of 0.06 to $0.15 \mathrm{~g} / \mathrm{g} \mathrm{m}$. $\mathrm{s}$. for the variety grain Oscar Blanco, for variety Noel Vietmeyer oscillates between 2.556 to $0.518 \mathrm{~kJ} / \mathrm{g}$ for moisture of 0.04 to $0.12 \mathrm{~g} / \mathrm{g} \mathrm{m}$.s., and for grain CICA of 4.645 to $0.176 \mathrm{~kJ} / \mathrm{g}$ for moisture of 0.06 to 0.14 $\mathrm{g} / \mathrm{g} \mathrm{m} . \mathrm{s}$, as for the flour of the variety Oscar Blanco is between 32.028 to $1.99 \mathrm{~kJ} / \mathrm{g}$ for moisture content

Cita: Choque-Quispe D, Ligarda-Samanez CA, Ramos-Pacheco BS, Taipe-Pardo F, Peralta-Guevara DE, Solano Reynoso AM. Evaluación de las isotermas de sorción de granos y harina de kiwicha (Amaranthus caudatus). rev.ion. 2018;31(2):67-81. doi:10.18273/revion.v31n2-2018005
\end{abstract}


of 0.05 to $0.12 \mathrm{~g} / \mathrm{g} \mathrm{m} . \mathrm{s}$, and for variety Noel Vietmeyer of 26.992 to $0.648 \mathrm{~kJ} / \mathrm{g}$ for moisture of de 0.06 to $0.20 \mathrm{~g} / \mathrm{g}$ m.s.

Keywords: Amaranth, adsorption, moisture of balance (Xe), heat isosteric.

\section{Resumo}

O objetivo principal foi avaliar as isotermas de sorção de grãos e farinha de amaranto (Amaranthus caudatus) das variedades Oscar Blanco, Noel Vietmeyer e CICA, utilizou-se o método estático gravimétrico para intervalos de humidade relativa de 10 a $90 \%$ a temperaturas de $18,20,25$ e $30{ }^{\circ} \mathrm{C}$. Estudaram-se 11 modelos matemáticos e modelaram-se a dados experimentais através de RNL tomando como critério de convergência o coeficiente de correlação $R^{2}$ e o erro médio relativo $\% E$, assim o modelo de Aldam e Shove apresento melhor ajuste para os grãos de amaranto, enquanto para a farinha foi o modelo de Peleg. Assim mesmo observou-se que a temperatura de armazenamento influi significativamente $(p$-value $<0,05)$ na humidade de equilíbrio $(X e)$ dos grãos e farinha de amaranto. O calor isostérico de sorção varia entre 6,340 a $0,235 \mathrm{KJ} / \mathrm{g}$ para humidades de 0,06 a $0,15 \mathrm{~g}$ de água/g m.s. para os grãos da variedade Oscar Blanco, para a variedade Noel Vietmeyer varia entre 2,556 a 0,518 KJ/g para humidades de 0,04 a 0,12 g de água/g m.s., e para o grão CICA de 4,645 a 0,176 KJ/g para humidades de 0,06 a $0,14 \mathrm{~g}$ de água/g m.s., enquanto para a farinha da variedade Oscar Blanco encontra-se entre 32,028 a $1,99 \mathrm{KJ} / \mathrm{g}$ para humidades de 0,05 a 0,12 $\mathrm{g}$ de água/g m.s., e para a variedade Noel Vietmeyer de 26,992 a $0,648 \mathrm{KJ} / \mathrm{g}$ para humidades de 0,06 a $0,20 \mathrm{~g}$ de água/g m.s.

Palavras-chave: Amaranto, adsorção, umidade de equilibrio (Xe), calor isostérico.

\section{Introducción}

La conservación de los cereales ha sido, es y será motivo de preocupación para el hombre, debido a la necesidad que tiene de preservarlo de factores físicos y microbiológicos del ambiente. Un reporte de la Organización de las Naciones Unidas para la Alimentación y la Agricultura FAO dice que el $30 \%$ de los cereales alimenticios post cosecha se pierde en los países del mundo en vías de desarrollo a causa de la manipulación inadecuada, deterioro y plagas [1,2]. En la provincia de Andahuaylas, este inconveniente no es ajeno, en especial en la zona rural donde los agricultores utilizan lugares poco apropiados para la conservación y almacenaje de los granos de kiwicha, sin el control adecuado de temperatura y humedad relativa del medio. Los cereales están constituidos principalmente de carbohidratos, que además contienen proteínas y lípidos en bajas concentraciones. El agua adsorbida en el producto se encuentra en equilibrio con el agua en estado de vapor en el aire de la atmósfera que lo rodea, por eso se suele llamar también humedad relativa en el equilibrio (\%HRE). Las isotermas de adsorción son el medio por el cual se evalúan las condiciones de almacenamiento de los granos de kiwicha y con ayuda de modelos matemáticos evaluar parámetros de humedad relativa y temperatura, de esta manera monitorear mejor su almacenamiento.

La actividad del agua es un concepto que mide el grado de libertad y/o actividad o disponibilidad en que se encuentra el agua contenida en los alimentos. Las sustancias solubles del alimento, como los azúcares y sales son las sustancias que ligan o atan el agua del alimento, haciéndole menos libre o disponible para el desarrollo de los microorganismos [3]

Si la humedad en el ambiente de un almacén excede el equilibrio de la humedad relativa (HRE) del alimento, el alimento absorbe la humedad del ambiente y viceversa [4, 5], el equilibrio entre un sólido húmedo y aire con cierto contenido en agua, se establece cuando la actividad del agua es igual a la humedad relativa de equilibrio del aire. Al cociente $p_{m}$ (presión de vapor del alimento) y $p^{v}$ (presión de vapor del agua pura) se le denomina actividad del agua $\left(a_{w}\right)$ y su valor se relaciona con determinados procesos de modificación o alteración de los productos alimentarios.

La actividad de agua está entre 0 y 1 , siendo más baja mientras más fuertemente ligada se encuentre el agua al material; tiende a la unidad cuando está tan débilmente adherida al material que su comportamiento se acerca al de su estado libre o puro [6].

La isoterma de adsorción se define como la relación entre la actividad de agua (o la humedad relativa de equilibrio de aire circundante) y el contenido de humedad de un material en equilibrio a temperatura constante, la cual se obtiene cuando el proceso de 
equilibrio parte de una muestra húmeda o seca, y a esta se le permite equilibrarse con la humedad del aire circundante perdiendo o ganando humedad, reflejando así, la forma como el agua se liga al sistema [7]. Existen diferentes tipos de isotermas de sorción en función del recubrimiento del adsorbente (capa monomolecular o multimolecular de adsorbato) dependiendo de la presencia de poros o capilares, mostrando todas ellas un cierto grado de histéresis [5].

Las isotermas de los alimentos, se dividen en tres regiones (Figura 1) denotadas por $A, B y$ $C$. En la región $A$, las moléculas de agua están enérgicamente ligadas a los sitios específicos en el sólido, estos sitios contienen a los grupos hidroxil de los polisacaridos, carbonil y amino de las proteínas, uniéndose a través de puentes de hidrogeno y fuerzas ión-dipolo. Es así que esta agua no está disponible como solvente y por lo tanto no colabora microbiológicamente, enzimáticamente o químicamente. A esta zona se la denomina monocapa [9]. En la región $B$ el agua se encuentra en multicapas [4], menos fuertemente unida, estando presente normalmente en pequeños capilares [10] y en la región $C$ se presenta estructuralmente y puede presentar soluciones [4], suelta en grandes capilares o está libre [10].

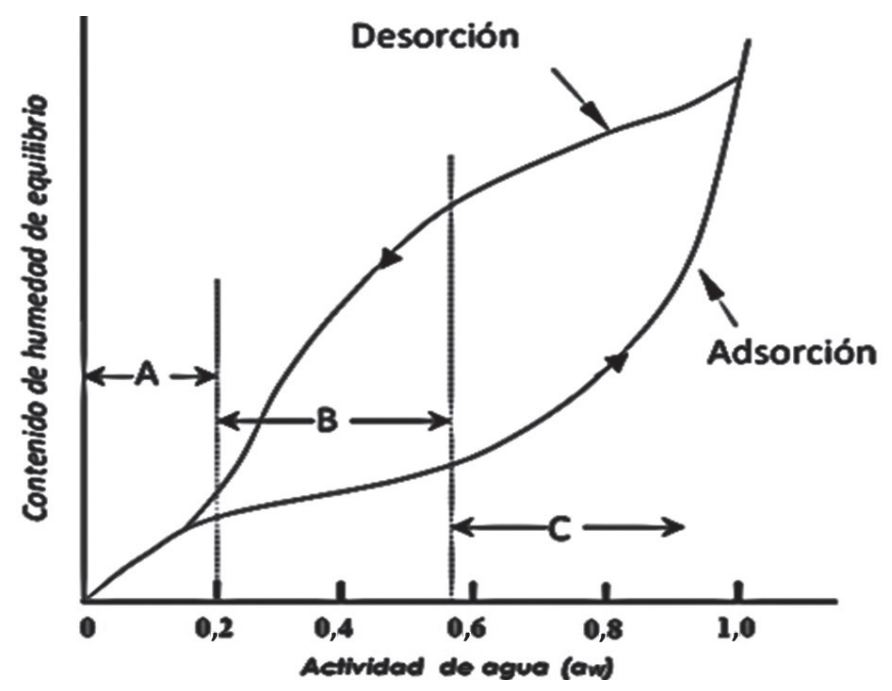

Figura 1. Isotermas de adsorción y desorción e histéresis [8].

Las isotermas de sorción de alimentos se construyen para conocer la actividad de agua de cada alimento a una determinada temperatura según su contenido en humedad [11].

Se han clasificado 77 modelos de isotermas considerando aspectos teóricos, semiempíricos y empíricos [35], aplicados para productos agrícolas y alimentos procesados [36], algunas de ellas basadas en el comportamiento termodinámico del sistema alimento-agua como BET y GAB [33], mientras que las empíricas y/o semi-empíricas, que se han propuesto para correlacionar el contenido de humedad de equilibrio con la actividad de agua de un alimento [34], el modelo de Oswin (Tabla 1) representa una expansión en serie para curvas en forma sigmoidea, propuesta para relacionar la humedad de diversos alimentos con actividad de agua alrededor de 0.5 [37], la ecuación semiempírica de Henderson es adecuada para alimentos con alto contenido de almidón [38], el modelo de Caurie principalmente utilizado para alimentos deshidratados, presenta el parámetro $X_{s}$ (contenido de humedad de seguridad) que indica el contenido de humedad al cual se lograría la máxima estabilidad del alimento durante su almacenamiento [39], en tanto la ecuación de Halsey permite explicar la condensación de las multicapas de agua principalmente en almidones $[39,40]$, el modelo semiempirico de Chung y Pfost aprobado por la Sociedad Americana de Ingenieros Agrícolas (ASAE) es recomendado generalmente para cereales y sus productos [42, 43], mientras que los modelos de Peleg y Adam y Shove son una aproximación empírica [41], encontrando utilidad por el rango de utilización de $a_{w}$ entre 0.0 a 1.0 [50]. 
Tabla 1. Ecuaciones para isotermas de sorción.

\begin{tabular}{|c|c|}
\hline Nombre del modelo & Modelo matemático \\
\hline GAB & $x_{e}=\frac{x_{m} c_{G A B} k_{G A B} a_{W}}{\left[\left(1-k_{G A B} a_{W}\right)\left(1-k_{G A B} a_{W}+c_{G A B} k_{G A B} a_{W}\right)\right]}$ \\
\hline BET & $x_{e}=\frac{x_{m} c_{B E T} a_{W}}{\left[\left(1-a_{W}\right)\left(1+\left(C_{B E T}-1\right) a_{W}\right)\right]}$ \\
\hline Oswin & $x_{e}=A\left[\frac{a_{W}}{1-a_{W}}\right]^{B}$ \\
\hline Henderson modificado & $1-a_{W}=\exp \left(-k T_{x}^{n}\right)$ \\
\hline Chung y Pfost & $a_{W}=\exp \left(\frac{A}{R T} \exp \left(-B x_{e}\right)\right)$ \\
\hline Adam y Shove & $x_{e}=a+b * a_{w}+c * a_{w}{ }^{2}+d * a_{w}{ }^{3}$ \\
\hline Hasley & $a_{w}=\exp \left[\frac{-A}{x_{e}^{B}}\right]$ \\
\hline Henderson & $1-a_{W}=\exp \left(-k x_{e}^{n}\right)$ \\
\hline Schuchmann, Peleg y Ray & $x_{e}=\frac{A * A_{m}}{\left(1+B * A_{m}\right) *\left(C-A_{m}\right)}$ \\
\hline Peleg & $X_{e}=A * a_{W}{ }^{c}+B * a_{W}{ }^{d} \quad c>1 \mathrm{y} d>1$ \\
\hline Caurie & $x_{e}=\exp \left[a_{w} \operatorname{In}(r)-\frac{1}{4,5 x_{s}}\right]$ \\
\hline
\end{tabular}

Donde: $A, B, C_{B E T} C, k_{G A B}, k, n, n ;, a, b, c, d$ son las constantes de las ecuaciones; $\mathrm{Xe}$, humedad de equilibrio expresada en base seca ( $g$ de agua/g masa seca); $X_{m}$, humedad de la monocapa molecular $(\mathrm{g} / \mathrm{g}$ m.s.); $R$, constante universal de los gases ideales; $\mathrm{T}$, Temperatura $(K), X_{S}$, contenido de humedad de seguridad que proporciona la máxima estabilidad al alimento deshidratado durante el almacenamiento $(\mathrm{g} / \mathrm{g}$ m.s), r, constante característica del material.

Para remover la humedad asociada a un material higroscópico, la energía necesaria es mayor que la utilizada para vaporizar igual cantidad de agua libre, en las mismas condiciones de presión y temperatura [12].

Iglesias y Chirife [13], presentan un método largamente usado para calcular el calor isostérico de sorción, sea para los procesos de adsorción o para desorción, y está dado por la Ecuación 1, ecuación de Clausius-Clayperon.

$$
q_{s t}=-R \frac{d \ln a_{W}}{d(1 / T)}
$$

Donde: $a_{w}$, actividad de agua, decimal; $T$, temperatura absoluta, $K ; q_{s t}$, calor isostérico neto de adsorción, $\mathrm{kJ} / \mathrm{kg}$; y $R$, constante universal de los gases, 8,314 $\mathrm{kJ} \mathrm{kmol}^{-1} \mathrm{~K}^{-1}$, para el vapor de agua $0,4619 \mathrm{~kJ} \mathrm{~kg}^{-1} \mathrm{~K}^{-1}$.
Para Tsami et al. [14] conocer el estado del agua en los productos alimenticios, plantea una relación exponencial empírica (Ecuación 2) entre el calor de sorción y el contenido de humedad de los alimentos deshidratados y frutas seca.

$$
q_{s t}=q_{0} \exp \left(X_{e} / X_{0}\right)
$$

Donde: $q_{s t}$, es el calor isostérico de adsorción neto, cuando el contenido de humedad es constante; $X_{e}$, es la humedad de equilibrio ( $g$ de agua/g muestra seca); $q_{0}$, es el calor isostérico de adsorción $(k J /$ $\mathrm{mol})$ de la primera molécula de agua en el alimento y se define cuando $X_{e} \rightarrow 0=>q_{s t} \rightarrow q_{0}, y X_{0}$ es el contenido de humedad característico para cada producto.

\section{Metodología experimental}

\section{Materia prima}

Los granos de kiwicha de las variedades en estudio fueron adquiridos del Centro Investigación en Cultivos Andinos - CICA de la la Facultad de Agronomía de la Universidad Nacional de San Antonio Abad del Cusco. La harina de kiwicha fue obtenida luego de la molienda y tamizado en una malla de 250 micras. 
Construcción de las isotermas de sorción

La construcción de las isotermas de adsorción, se basó en el método estático gravimétrico o método de control continuo de cambios de peso, descrito por [15]. Se prepararon soluciones saturadas de acuerdo a las sustancias químicas, que se presentan en la Tabla 2, cada una presenta una $H R E$ o $a_{w}$ a la temperatura deseada.

Tabla 2. Sustancias químicas y sus ecuaciones para obtener $a_{w}$ a la temperatura deseada.

\begin{tabular}{ccc}
\hline Sustancia química & Ecuación & \\
\hline $\mathrm{LiCl}$ & In $\left.a_{w}=(500,95 / T)\right)-3,85 ; R^{2}=0,998$ & {$[15]$} \\
$\mathrm{KC}_{2} \mathrm{H}_{3} \mathrm{O}_{2}$ & In $\left.a_{w}=(961,39 / T)\right)-4,33 ; R^{2}=0,98$ & {$[15]$} \\
$\mathrm{MgCl}$ & $a_{w}=0,365-2,532 \times 10^{-3} T+5,071 \times 10^{-5} T^{2}-$ & {$[15]$} \\
$\mathrm{Mg}\left(\mathrm{NO}_{3}\right)_{2}$ & $4,166 \times 10^{-7} T^{3} ; R^{2}=0,963$ & {$[15]$} \\
$\mathrm{KI}$ & In $\left.a_{w}=(356,60 / T)\right)-1,82 ; R^{2}=0,99$ & {$[15]$} \\
$\mathrm{NaCl}$ & In $\left.a_{w}=(255,90 / T)\right)-1,23 ; R^{2}=1,00$ & {$[15]$} \\
$\mathrm{KCl}$ & In $\left.a_{w}=(228,92 / T)\right)-1,04 ; R^{2}=0,96$ & {$[15]$} \\
$\mathrm{BaCl}_{2}$ & In $\left.a_{w}=(367,58 / T)\right)-1,39 ; R^{2}=0,97$ & {$[32]$} \\
\hline
\end{tabular}

Las isotermas de adsorción se evaluaron a las temperaturas de $18^{\circ} \mathrm{C}, 20^{\circ} \mathrm{C}, 25^{\circ} \mathrm{C}$ y $30^{\circ} \mathrm{C}$, a fin de determinar la humedad de equilibrio $\left(X_{e}\right)$ para los granos de kiwicha.

\section{Determinación de humedad de equilibrio $\left(x_{\mathrm{e}}\right)$}

La humedad de equilibrio $\left(X_{e}\right)$ fue calculada por la diferencia entre la masa de la muestra que presentó equilibrio y la masa seca (Ecuación 3) [16].

$$
x_{e}=\frac{m_{e q}-m_{s}}{m_{s}}
$$

Donde: $X_{e}=$ humedad de equilibrio en b. s.; $m_{e q}$ = masa de la muestra en el equilibrio, $(g)$., y $m_{s}=$ masa de la muestra seca, $(g)$.

\section{Estimación y modelamiento de las isotermas}

La calidad del ajuste de los modelos propuestos se evaluó por medio del coeficiente de determinación $\left(R^{2}\right)$, para conseguir un buen modelado de los datos experimentales y por el porcentaje de error medio relativo $(\% E)$ parámetro estadístico ampliamente utilizado en isotermas de alimentos (Ecuación 4) [17].

$$
\text { (\%) } E=\frac{100}{n} \sum_{i=1}^{n} \frac{X_{e i}-X_{c i}}{X_{e i}}
$$

Donde: $X_{e i}$, es el contenido de humedad experimental (g agua/ g m.s.); $X_{c i}$, es el contenido de humedad calculada a partir de cada modelo ( $\mathrm{g}$ agua/g m.s.); $n$, es el número de observaciones.
El ajuste de las isotermas, se realizó a través del método de estimación matemática Quasi-Newton, ya que presenta los siguientes criterios: Valor más bajo de la diferencia de los mínimos cuadrados, y valor más alto del coeficiente de determinación $R^{2}$.

\section{Evaluación de la monocapa GAB}

El valor de la monocapa $X_{m}$, se determinó, a través de la isoterma $G A B$ ajustada, para las temperaturas de $18^{\circ} \mathrm{C}, 20^{\circ} \mathrm{C}, 25^{\circ} \mathrm{C}$ y $30^{\circ} \mathrm{C}$.

\section{Determinación del calor isostérico $\boldsymbol{q}_{s t}$}

Se graficó In $a_{W} v s 1 / T$, a sus respectivas humedades de equilibrio, siendo la pendiente $-q_{s t} / R$ [18].

Así mismo los valor de $a_{w}$ y humedad de equilibrio fueron obtenidos a partir de la isoterma que presenta mejor ajuste y describe mejor a los datos experimentales (isoterma de adsorción), para el intervalo comprendido entre 0,05 a 0,25 de $X_{e}$. Por otra parte, los datos de $q_{s t}$ referidos a $X_{e}$ correspondientes, se ajustaron a la ecuación de Tsami.

\section{Resultados y discusión}

\section{Humedad de equilibrio}

En la Figura 2, se observa el comportamiento de la isoterma de adsorción de humedad del grano de kiwicha de la variedad Oscar Blanco para cada temperatura de estudio, se aprecia que ésta corresponde a una isoterma del tipo II, asimismo 
se puede ver que existe un entrecruzamiento de las isotermas a HRE de 0,68 o $68 \%$, mientras que para la variedad Noel Vietmeyer se muestra en las figuras 3 y 4.

Shafiur [19], manifiesta que el entrecruzamiento se debe a que en algunos alimentos una parte del soluto (sales y/o azúcar), está ligada a un polímero (almidón y proteína) y la otra parte es cristalina o amorfa, de otra parte se puede deber a la composición de los granos, sobre todo en la superficie referidos a la fibra, grasas (como ceras) y carbohidratos, de hecho la variedad Oscar Blanco presenta un contenido mayor de fibra de 5,04\% y $0,36 \%$ de grasa, mientras que la variedad Noel Vietmeyer $3,26 \%$ de fibra y $0,24 \%$ de grasa [21], otro factor es la desnaturalización de proteínas, que produce un desdoblamiento de la molécula exponiendo un mayor número de sitios polares disponibles para la unión de agua [20], y estos pseudo cereales son ricos en contenidos proteicos.

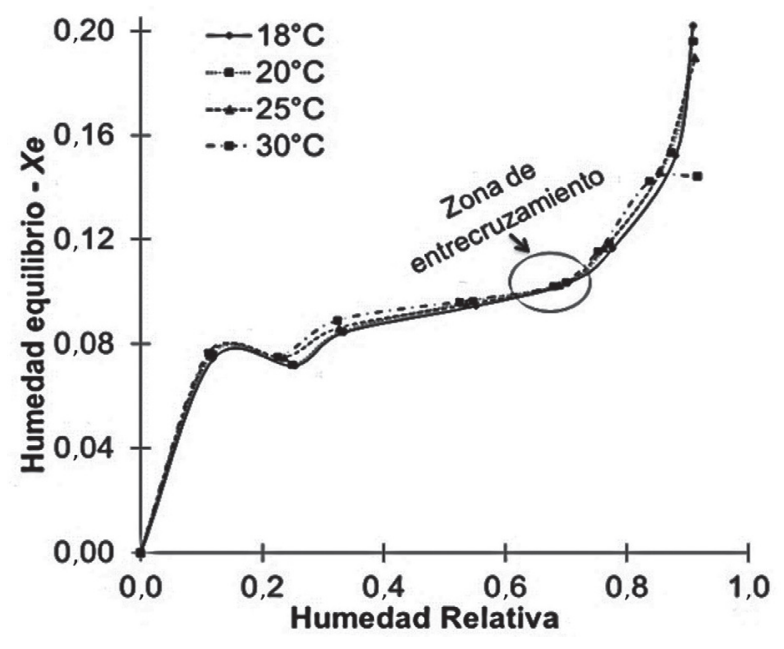

Figura 2. Isotermas de adsorción del grano de kiwicha variedad Oscar Blanco.

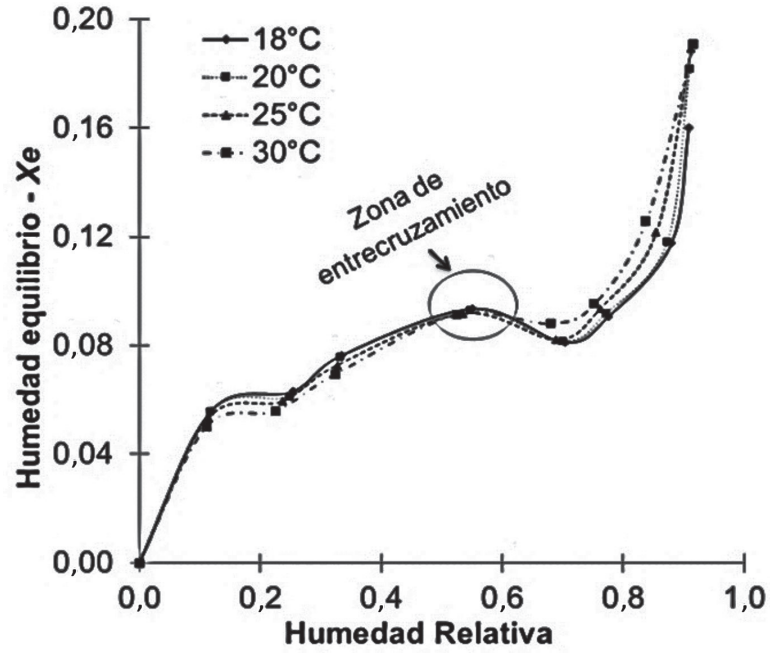

Figura 3. Isotermas de adsorción del grano de kiwicha variedad Noel Vietmeyer.

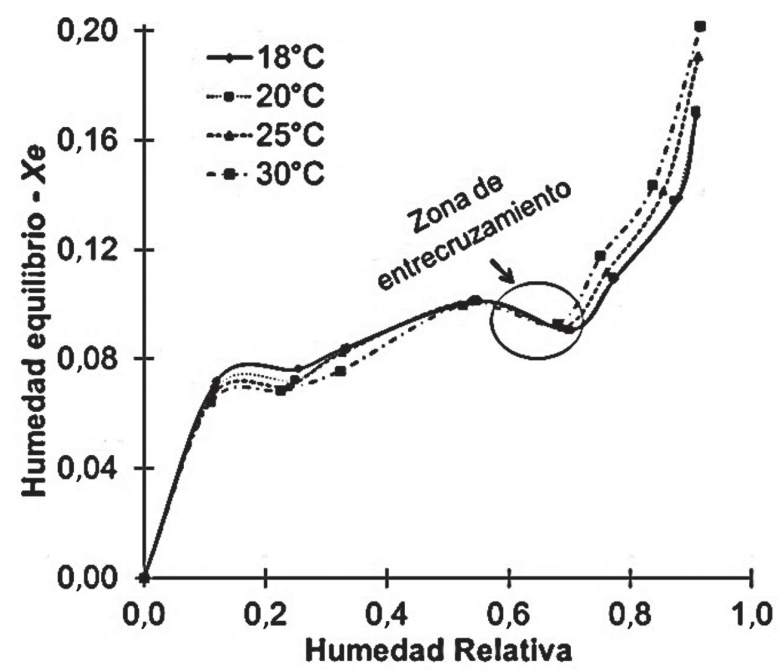

Figura 4. Isotermas de adsorción del grano de kiwicha variedad CICA. 


\section{Influencia de la temperatura en la humedad equilibrio}

El efecto de la influencia de la temperatura en el comportamiento higroscópico de los cereales estudiados, se manifiesta antes y después de la zona de entrecruzamiento, ocurriendo la reversión de la tendencia de las curvas, es decir $X_{e}$ es menor antes del entrecruzamiento y se incrementa pasado este zona, y con el aumento de la temperatura, lo que indica que cualquier cambio de temperatura influenciará significativamente ( $p$-value $<0,05)$ en la humedad de equilibrio de los granos de kiwicha de las variedades Noel Vietmeyer y CICA, aunque este hecho no es significativo ( $p$-value $>0,05$ ) para la variedad Oscar Blanco, esto debido al alto contenido de fibra, en comparación a las otras variedades.
Modelamiento de las isotermas de adsorción Al evaluar los modelos propuestos, se observó que el modelo de Adam y Shove se ajustó adecuadamente con \%E $<7,3$ y $\mathrm{R}^{2}>0,94$ para la variedad Oscar Blanco, \%E < 12,3 y $\mathrm{R}^{2}>0,93$ para Noel Vietmeyer, y para la variedad CICA \%E $<7,9$ y $R^{2}>0,97$, asimismo el modelo de Halsey presentó buen ajuste con valores de \%E $<8,7$ y $R^{2}>0,89$ para la variedad Oscar Blanco, \%E < 13,7 y $R^{2}>0,91$ para Noel Vietmeyer, y para la variedad $\mathrm{CICA} \% \mathrm{E}<9,7 \mathrm{yR}^{2}>0,95$ (Tabla 3 ), es así que estos dos modelos permitirían describir adecuadamente el comportamiento higroscópico de la kiwicha a las temperaturas de estudio, no obstante los otros modelos presentaron valores de $R^{2}$ y \%E alentadores y aceptables pues en la mayoría de los casos presentan $R^{2}>0,7$.

Tabla 3. Estadígrafos de las isotermas para las variedades de granos de kiwicha.

\begin{tabular}{|c|c|c|c|c|c|c|c|c|c|c|c|c|c|}
\hline \multirow{2}{*}{ Modelo } & & \multicolumn{4}{|c|}{ Variedad Oscar Blanco } & \multicolumn{4}{|c|}{ Variedad Noel Vietmeyer } & \multicolumn{4}{|c|}{ Variedad CICA } \\
\hline & & $18^{\circ} \mathrm{C}$ & $20^{\circ} \mathrm{C}$ & $25^{\circ} \mathrm{C}$ & $30^{\circ} \mathrm{C}$ & $18^{\circ} \mathrm{C}$ & $20^{\circ} \mathrm{C}$ & $25^{\circ} \mathrm{C}$ & $30^{\circ} \mathrm{C}$ & $18^{\circ} \mathrm{C}$ & $20^{\circ} \mathrm{C}$ & $25^{\circ} \mathrm{C}$ & $30^{\circ} \mathrm{C}$ \\
\hline \multirow{2}{*}{ GAB } & $\% \mathrm{E}$ & 11,2 & 22,9 & 9,3 & 4,8 & 12,8 & 16,5 & 16,3 & 12,2 & 10,2 & 10,1 & 12,0 & 10,8 \\
\hline & $R^{2}$ & 0,88 & 0,52 & 0,91 & 0,94 & 0,62 & 0,88 & 0,92 & 0,75 & 0,92 & 0,93 & 0,94 & 0,97 \\
\hline \multirow{2}{*}{ Oswin } & $\% \mathrm{E}$ & 11,3 & 9,7 & 9,3 & 5,5 & 11,9 & 15,5 & 15,8 & 12,6 & 9,0 & 9,0 & 11,0 & 10,4 \\
\hline & $R^{2}$ & 0,88 & 0,90 & 0,90 & 0,93 & 0,91 & 0,89 & 0,92 & 0,92 & 0,93 & 0,94 & 0,94 & 0,96 \\
\hline \multirow{2}{*}{$\begin{array}{l}\text { Henderson } \\
\text { modificado }\end{array}$} & $\% \mathrm{E}$ & 14,3 & 12,8 & 12,2 & 7,3 & 13,1 & 17,0 & 18,3 & 16,7 & 10,3 & 10,2 & 13,0 & 13,7 \\
\hline & $R^{2}$ & 0,79 & 0,81 & 0,80 & 0,89 & 0,87 & 0,84 & 0,87 & 0,87 & 0,88 & 0,90 & 0,89 & 0,92 \\
\hline \multirow{2}{*}{$\begin{array}{c}\text { Chung y } \\
\text { Pfost }\end{array}$} & $\% \mathrm{E}$ & 11,5 & 10,3 & 9,2 & 5,3 & 11,5 & 14,5 & 14,8 & 12,8 & 8,5 & 8,7 & 10,4 & 10,4 \\
\hline & $R^{2}$ & 0,85 & 0,87 & 0,88 & 0,93 & 0,90 & 0,87 & 0,90 & 0,90 & 0,92 & 0,93 & 0,93 & 0,94 \\
\hline \multirow{2}{*}{$\begin{array}{l}\text { Adam y } \\
\text { Shove }\end{array}$} & $\% \mathrm{E}$ & 7,3 & 6,4 & 4,8 & 4,3 & 10,2 & 12,3 & 11,4 & 10,2 & 6,8 & 7,2 & 7,9 & 7,2 \\
\hline & $R^{2}$ & 0,94 & 0,96 & 0,98 & 0,96 & 0,94 & 0,93 & 0,96 & 0,96 & 0,97 & 0,97 & 0,97 & 0,98 \\
\hline \multirow{2}{*}{ Halsey } & $\% \mathrm{E}$ & 8,7 & 7,3 & 6,7 & 4,5 & 10,4 & 13,7 & 13,1 & 9,1 & 9,7 & 7,6 & 8,9 & 7,4 \\
\hline & $R^{2}$ & 0,92 & 0,94 & 0,95 & 0,89 & 0,92 & 0,91 & 0,95 & 0,95 & 0,95 & 0,96 & 0,97 & 0,98 \\
\hline \multirow{2}{*}{ Henderson } & $\% \mathrm{E}$ & 14,3 & 12,8 & 12,3 & 7,3 & 13,1 & 17,0 & 18,3 & 16,7 & 10,3 & 10,2 & 13,0 & 13,7 \\
\hline & $R^{2}$ & 0,79 & 0,81 & 0,80 & 0,89 & 0,87 & 0,84 & 0,87 & 0,87 & 0,88 & 0,90 & 0,89 & 0,92 \\
\hline \multirow{2}{*}{ Schufman } & $\% \mathrm{E}$ & 17,3 & 8,0 & 7,5 & 4,8 & 13,4 & 16,2 & 15,1 & 11,4 & 9,9 & 9,7 & 10,8 & 9,0 \\
\hline & $R^{2}$ & 0,81 & 0,94 & 0,95 & 0,94 & 0,90 & 0,89 & 0,93 & 0,93 & 0,93 & 0,94 & 0,95 & 0,98 \\
\hline \multirow{2}{*}{ Peleg } & $\% \mathrm{E}$ & 16,5 & 3,9 & 14,8 & 10,1 & 4,2 & 17,2 & 19,1 & 4,3 & 4,0 & 4,3 & 4,2 & 4,8 \\
\hline & $R^{2}$ & 0,65 & 0,99 & 0,66 & 0,79 & 0,80 & 0,77 & 0,79 & 0,79 & 0,81 & 0,83 & 0,82 & 0,84 \\
\hline \multirow{2}{*}{ Caurie } & $\% \mathrm{E}$ & 13,7 & 12,2 & 11,7 & 6,2 & 13,0 & 16,7 & 17,8 & 16,1 & 10,2 & 10,4 & 13,0 & 13,6 \\
\hline & $R^{2}$ & 0,80 & 0,83 & 0,83 & 0,92 & 0,86 & 0,84 & 0,86 & 0,86 & 0,89 & 0,90 & 0,90 & 0,85 \\
\hline
\end{tabular}

En las Figuras 5, 6 y 7 se presenta las isotermas ajustadas para los modelos de Adam y Shove (A\&S) y Halsey, observándose ligera desviación una de otra, no obstante de acuerdo a los valores de $R^{2}$ y $\% E$ el modelo de A\&S se ajusta adecuadamente para las tres variedades de kiwicha a las temperaturas de estudio, cuyo valor del parámetro A se incrementa con la temperatura para la variedad Oscar Blanco (Tabla 4), mientras que para las variedades Noel Vietmeyer y CICA sucede lo contrario; los otros parámetros varían aleatoriamente, aunque no tiene ningún indicativo termodinámico este comportamiento, puesto que se trata de una ecuación eminentemente empírica. 
rev.ion. 2018;31(2):67-81. Bucaramanga (Colombia).

Tabla 4. Parámetros de las isotermas para las variedades de granos de kiwicha.

\begin{tabular}{|c|c|c|c|c|c|c|c|c|c|c|c|c|c|}
\hline \multirow{2}{*}{\multicolumn{2}{|c|}{ Modelo }} & \multicolumn{4}{|c|}{ Variedad Oscar Blanco } & \multicolumn{4}{|c|}{ Variedad Noel Vietmeyer } & \multicolumn{4}{|c|}{ Variedad CICA } \\
\hline & & $18^{\circ} \mathrm{C}$ & $20^{\circ} \mathrm{C}$ & $25^{\circ} \mathrm{C}$ & $30^{\circ} \mathrm{C}$ & $18^{\circ} \mathrm{C}$ & $20^{\circ} \mathrm{C}$ & $25^{\circ} \mathrm{C}$ & $30^{\circ} \mathrm{C}$ & $18^{\circ} \mathrm{C}$ & $20^{\circ} \mathrm{C}$ & $25^{\circ} \mathrm{C}$ & $30^{\circ} \mathrm{C}$ \\
\hline \multirow{3}{*}{ GAB } & $X m$ & 0,054 & 0,056 & 0,058 & 0,067 & 0,050 & 0,045 & 0,042 & 0,042 & 0,060 & 0,058 & 0,053 & 0,051 \\
\hline & $C_{g a b}$ & $8,1 \mathrm{E} 5$ & 7,134 & 1,7E6 & $6,5 \mathrm{E} 5$ & $4,4 \mathrm{E} 7$ & $7,1 \mathrm{E} 6$ & $8,5 \mathrm{E} 5$ & $1,4 \mathrm{E} 6$ & $2,6 \mathrm{E} 6$ & 1,3E6 & $3,8 \mathrm{E} 6$ & $3,8 \mathrm{E} 9$ \\
\hline & $K_{\text {gab }}$ & 0,766 & 0,752 & 0,730 & 0,586 & 0,702 & 0,775 & 0,823 & 0,836 & 0,664 & 0,685 & 0,759 & 0,801 \\
\hline \multirow{4}{*}{$\begin{array}{c}\text { Adam y } \\
\text { Shove }\end{array}$} & $A$ & 0,040 & 0,045 & 0,046 & 0,068 & 0,006 & $-0,004$ & $-0,007$ & $-0,009$ & 0,035 & 0,030 & 0,021 & 0,020 \\
\hline & $B$ & 0,348 & 0,309 & 0,307 & 0,076 & 0,502 & 0,596 & 0,619 & 0,552 & 0,377 & 0,390 & 0,465 & 0,410 \\
\hline & $C$ & $-0,918$ & $-0,817$ & $-0,801$ & $-0,136$ & $-1,130$ & $-1,395$ & $-1,474$ & $-1,291$ & $-0,899$ & $-0,910$ & $-1,118$ & $-1,006$ \\
\hline & $D$ & 0,782 & 0,710 & 0,689 & 0,162 & 0,820 & 1,032 & 1,111 & 0,992 & 0,699 & 0,705 & 0,879 & 0,832 \\
\hline \multirow{2}{*}{ Halsey } & $A$ & 0,001 & 0,001 & 0,000 & 0,000 & 0,000 & 0,001 & 0,001 & 0,002 & 0,000 & 0,000 & 0,001 & 0,001 \\
\hline & $B$ & 2,812 & 2,895 & 3,090 & 4,525 & 3,265 & 2,754 & 2,461 & 2,375 & 3,279 & 3,432 & 2,886 & 2,617 \\
\hline
\end{tabular}

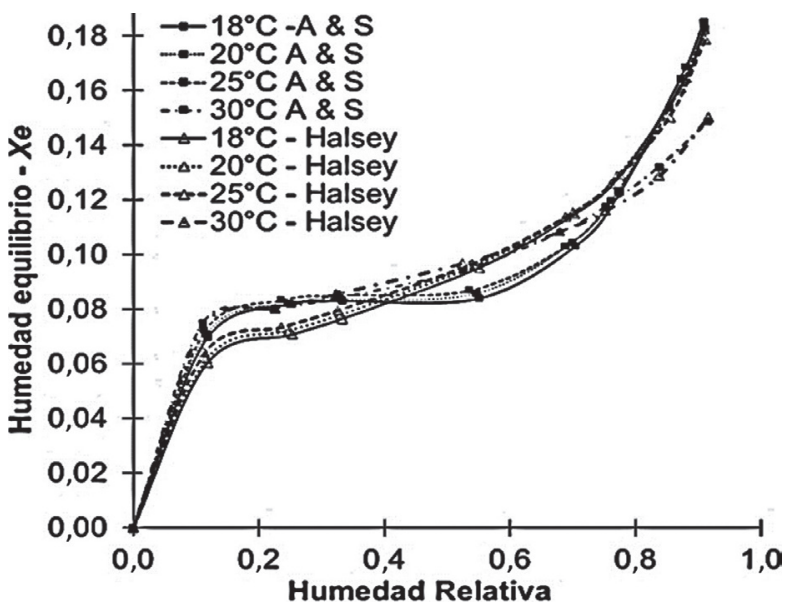

Figura 5. Isotermas de adsorción ajustada del grano de kiwicha Oscar Blanco.

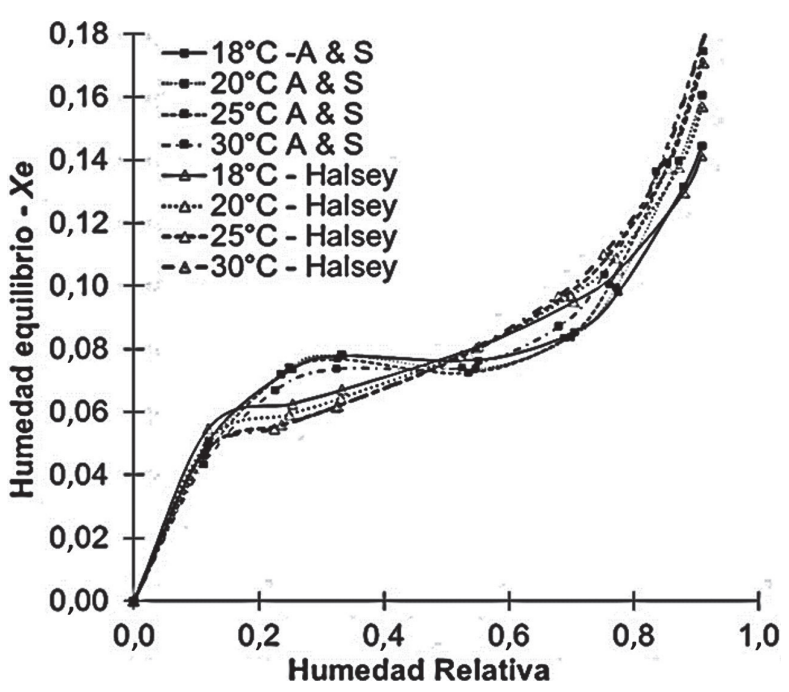

Figura 6. Isotermas de adsorción ajustada del grano de kiwicha Noel Vietmeyer.

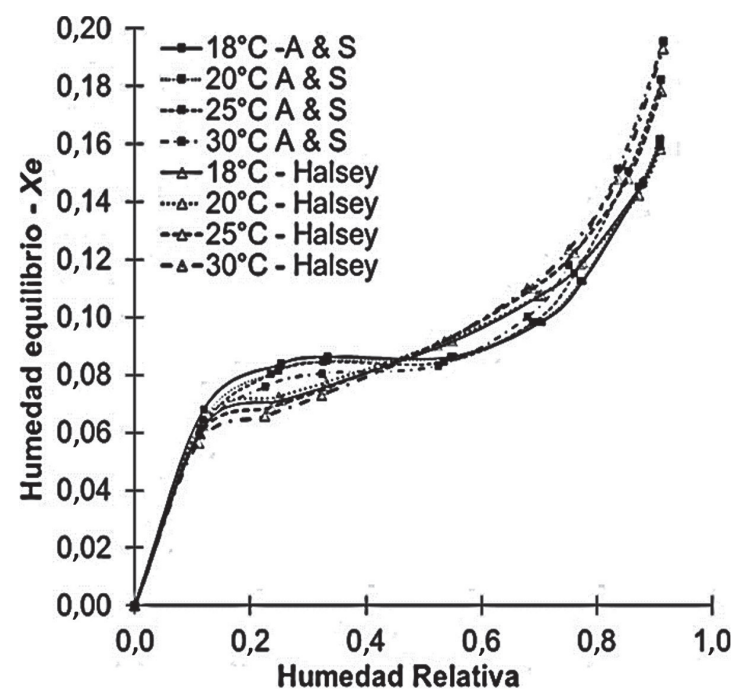

Figura 7. Isotermas de adsorción ajustada del grano de kiwicha CICA. 
De otra parte para la harina de kiwicha de las variedades Oscar Blanco y Noel Vietmeyer, los modelos de isotermas estudiados presentaron buen ajuste, con valores de $R^{2}>0,7$ y aunque $\% \mathrm{E}$ es considerablemente elevado para algunos casos, no obstante el modelo de Peleg presentó valores de $R^{2}$ cercanos a la unidad y $\% \mathrm{E}<4,3$ (Tabla 5), Pumacahua-Ramos et al. [44] mostro que el modelo de Peleg se ajustó adecuadamente para harina de quinua (Chopodium quinoa) aunque con valores de \%E mayores al reportado. En la Figura 8 se observa las curvas de adsorción para la harina de kiwicha de la variedad Noel Vietmeyer, ajustadas a través del modelo Peleg, estas se superponen casi completamente a los datos experimentales en todos los rangos de temperatura. Asimismo en la Tabla 6 se presenta los parámetros del modelo Peleg, PumacahuaRamos et al. [44] encontró similares resultado para la harina de quinua (Chenopodium quinoa), con tendencias de disminución del parámetro $\mathrm{A}$ y $B$ a medida que se incrementa la temperatura, este comportamiento es similar en esta investigación.

Tabla 5. Estadígrafos de las isotermas para las variedades de harina de kiwicha.

\begin{tabular}{|c|c|c|c|c|c|c|c|c|c|}
\hline \multicolumn{2}{|c|}{ Modelo } & \multicolumn{4}{|c|}{ Variedad Oscar Blanco } & \multicolumn{4}{|c|}{ Variedad Noel Vietmeyer } \\
\hline 1010 & & $18^{\circ} \mathrm{C}$ & $20^{\circ} \mathrm{C}$ & $25^{\circ} \mathrm{C}$ & $30^{\circ} \mathrm{C}$ & $18^{\circ} \mathrm{C}$ & $20^{\circ} \mathrm{C}$ & $25^{\circ} \mathrm{C}$ & $30^{\circ} \mathrm{C}$ \\
\hline \multirow{2}{*}{ GAB } & $\% \mathrm{E}$ & 7,7 & 10,0 & 6,0 & 4,8 & 12,9 & 13,1 & 13,7 & 13,0 \\
\hline & $R^{2}$ & 0,86 & 0,79 & 0,92 & 0,94 & 0,83 & 0,85 & 0,91 & 0,95 \\
\hline \multirow{2}{*}{ Oswin } & $\% \mathrm{E}$ & 7,2 & 9,7 & 5,3 & 5,5 & 12,3 & 12,5 & 13,6 & 13,6 \\
\hline & $R^{2}$ & 0,90 & 0,81 & 0,93 & 0,93 & 0,85 & 0,87 & 0,91 & 0,94 \\
\hline \multirow{2}{*}{$\begin{array}{l}\text { Henderson } \\
\text { modificado }\end{array}$} & $\% \mathrm{E}$ & 8,9 & 12,8 & 7,5 & 7,3 & 13,4 & 13,9 & 16,2 & 17,9 \\
\hline & $R^{2}$ & 0,80 & 0,81 & 0,85 & 0,89 & 0,79 & 0,81 & 0,84 & 0,88 \\
\hline \multirow{2}{*}{ Chung y Pfost } & $\% \mathrm{E}$ & 6,9 & 10,3 & 5,0 & 5,3 & 11,6 & 11,9 & 13,2 & 14,2 \\
\hline & $R^{2}$ & 0,88 & 0,79 & 0,94 & 0,93 & 0,85 & 0,86 & 0,89 & 0,92 \\
\hline \multirow{2}{*}{ Adam y Shove } & $\% \mathrm{E}$ & 4,2 & 10,8 & 1,7 & 4,3 & 6,3 & 6,1 & 5,0 & 5,0 \\
\hline & $R^{2}$ & 0,96 & 0,90 & 0,98 & 0,96 & 0,96 & 0,97 & 0,99 & 0,99 \\
\hline \multirow{2}{*}{ Halsey } & $\% \mathrm{E}$ & 6,3 & 7,2 & 3,6 & 4,5 & 10,8 & 10,8 & 10,9 & 10,0 \\
\hline & $R^{2}$ & 0,92 & 0,94 & 0,97 & 0,89 & 0,88 & 0,91 & 0,94 & 0,97 \\
\hline \multirow{2}{*}{ Henderson } & $\% \mathrm{E}$ & 8,9 & 12,8 & 7,5 & 7,3 & 13,4 & 13,9 & 16,2 & 17,9 \\
\hline & $R^{2}$ & 0,80 & 0,81 & 0,85 & 0,89 & 0,79 & 0,81 & 0,84 & 0,88 \\
\hline \multirow{2}{*}{ Schufman } & $\% \mathrm{E}$ & 8,1 & 10,0 & 5,5 & 4,1 & 12,5 & 12,7 & 12,6 & 10,6 \\
\hline & $R^{2}$ & 0,87 & 0,82 & 0,94 & 0,95 & 0,86 & 0,88 & 0,93 & 0,97 \\
\hline \multirow{2}{*}{ Peleg } & $\% \mathrm{E}$ & 0,7 & 3,9 & 0,5 & 4,3 & 1,4 & 1,1 & 1,8 & 3,7 \\
\hline & $R^{2}$ & 1,00 & 1,00 & 1,00 & 0,96 & 0,70 & 0,72 & 1,00 & 0,79 \\
\hline \multirow{2}{*}{ Caurie } & $\% \mathrm{E}$ & 8,5 & 12,2 & 6,9 & 6,2 & 13,1 & 13,5 & 15,5 & 16,5 \\
\hline & $R^{2}$ & 0,82 & 0,72 & 0,87 & 0,92 & 0,79 & 0,81 & 0,85 & 0,89 \\
\hline
\end{tabular}


rev.ion. 2018;31(2):67-81. Bucaramanga (Colombia).

Tabla 6. Parámetros de las isotermas de las variedades de harina de kiwicha.

\begin{tabular}{|c|c|c|c|c|c|c|c|c|c|}
\hline \multirow{2}{*}{\multicolumn{2}{|c|}{ Modelo }} & \multicolumn{4}{|c|}{ Variedad Oscar Blanco } & \multicolumn{4}{|c|}{ Variedad Noel Vietmeyer } \\
\hline & & $18^{\circ} \mathrm{C}$ & $20^{\circ} \mathrm{C}$ & $25^{\circ} \mathrm{C}$ & $30^{\circ} \mathrm{C}$ & $18^{\circ} \mathrm{C}$ & $20^{\circ} \mathrm{C}$ & $25^{\circ} \mathrm{C}$ & $30^{\circ} \mathrm{C}$ \\
\hline \multirow{3}{*}{ GAB } & $X m$ & 0,109 & 0,056 & 0,109 & 6,7E-02 & 0,084 & 0,081 & 0,073 & $6,7 \mathrm{E}-02$ \\
\hline & $C_{g a b}$ & $5,3 \mathrm{E}+06$ & $5,6 \mathrm{E}+09$ & $1,7 \mathrm{E}+06$ & $1,2 \mathrm{E}+10$ & $4,4 \mathrm{E}+07$ & $1,6 \mathrm{E}+06$ & $6,8 \mathrm{E}+09$ & $6,7 \mathrm{E}+08$ \\
\hline & $K_{g a b}$ & 0,601 & 0,752 & 0,578 & 0,586 & 0,601 & 0,639 & 0,740 & 0,807 \\
\hline \multirow{4}{*}{ Peleg } & A & 0,764 & 0,291 & 0,184 & 0,080 & 0,130 & 0,705 & 0,130 & 0,318 \\
\hline & B & 0,183 & 0,180 & 0,166 & 0,090 & 1.334 & 0,431 & 0,381 & 0,125 \\
\hline & c & 22,168 & 12,532 & 8,369 & 3,491 & 0,111 & 20,444 & 0,134 & 8,283 \\
\hline & d & 0,191 & 0,206 & 0,139 & 0,084 & 27,580 & 0,120 & 11,951 & 0,142 \\
\hline
\end{tabular}

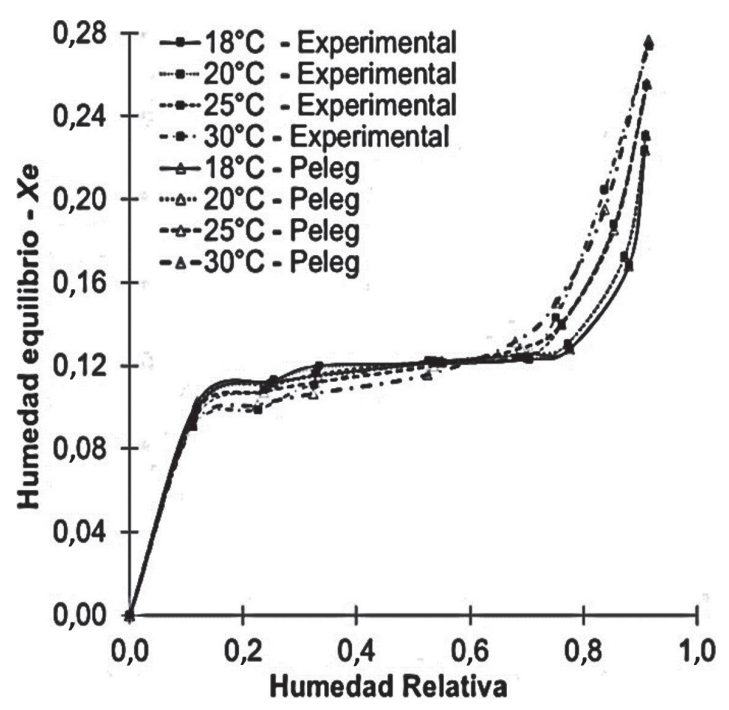

Figura 8. Isotermas de adsorción de la harina kiwicha Noel Vietmeyer.

Evaluación de la monocapa de GAB y parámetros fisicoquímicos

El valor de la monocapa de $G A B\left(X_{m}\right)$ que se presentan en las Tablas 4 y 6 para el grano de kiwicha de la variedad Oscar Blanco, oscila entre 0,0541 a $0,0673 \mathrm{~g} \mathrm{H}_{2} \mathrm{O} / g$ m.s., el valor de la monocapa es directamente proporcional con la temperatura (relación lineal de $X_{m}$ con la temperatura).

Choque et al. [22], reportan valores de monocapa $\left(X_{G A B}\right)$ de quinua (Chenopodium quinoa) variedad blanco de Junín, que oscilan entre 0,037 a 0,031 g de agua de equilibrio/g m.s., para las temperaturas de $20^{\circ} \mathrm{C}$ a $35^{\circ} \mathrm{C}$. Choque [23] presenta valores de monocapa de $G A B$ para el maíz morado (Zea mays L.) de $0,076,0,068$ y $0,064 \mathrm{~g}$ agua/g m.s. para las temperaturas de 18,25 y $35^{\circ} \mathrm{C}$.

Brett et al. [24], menciona que el cereal durante el primer nivel de adsorción de agua; a bajas $a_{w}$, el agua forma una monocapa que se fija sobre grupos polares de alta energía de enlace (del tipo - $\mathrm{CO}$, $-\mathrm{COO}-\mathrm{y}-\mathrm{NH}^{3+}$ ) de los constituyentes hidrofílicos (almidones, proteínas y pentosas) de los cereales, mediante atracciones electrostáticas, enlaces dipolares y enlaces de hidrógenos.

La presencia de poros y capilares en un determinado absorbente (en este caso el grano de kiwicha variedad Oscar Blanco), influye de manera significativa en la humedad de monocapa; el incremento en la temperatura determina la disminución en la higroscopicidad del alimento con excepción de ciertos azúcares y componentes alimenticios de bajo peso molecular [25].

Mientras que en los granos de kiwicha de la variedad Noel Vietmeyer y CICA la humedad de monocapa $X_{m}$ desciende debido a que a una mayor temperatura la monocapa se condensa de la superficie del grano, ya que presenta menor cantidad de compuestos polares disponibles para enlazarse y puede que también sea por el tipo de enlace que haya sucedido entre el adsorbente y las moléculas de agua.

Los parámetros fisicoquímicos de la isoterma de $\operatorname{GAB}\left(X_{m}, C_{G A B}\right.$ y $\left.k_{G A B}\right)$, cabe mencionar que $C_{G A B}$ es la constante de Guggenheim, $k$ una constante correctiva que describe las propiedades de adsorción de agua más allá de la monocapa y $X_{m}$ es la ganancia del agua en la monocapa $(g / 100 \mathrm{~g}$ la materia seca). Los 3 parámetros $\left(X_{m}, C_{G A B}\right.$ y $\left.k_{G A B}\right)$ caracterizan las interacciones del agua con los macroconstituyentes del alimento [26, 29].

Las constantes $C_{G A B}$ (constante energética) y $k_{G A B}$ están relacionadas a la energía de interacción entre la primera capa de moléculas (monocapa) y las capas distantes de moléculas de agua adsorbidas (comportamiento líquido). Las mismas dependen de la temperatura y dicha dependencia puede ser expresada por una ecuación del tipo de Arrhenius [27], Prieto et al. [28], indica que $C_{G A B}$ es una constante, $k$ una constante correctiva que describe las propiedades de adsorción de agua más allá de la monocapa. 
Evaluación del calor isostérico de adsorción

El calor isostérico de sorción es un parámetro que permite extraer información de la energía necesaria para alcanzar un determinado contenido en humedad durante el proceso de adsorción [30]. Para el cálculo del calor isostérico de sorción, $q_{s t}$, de los granos kiwicha, se consideraron los valores de $X_{e}$ especificados en las Tablas 7 y 8 a las temperaturas de evaluación, $X_{e}$ fue determinado con el modelo de Adam y Shove para los granos de kiwicha mientras que para sus harinas se utilizó el modelo Peleg.

Tabla 7. Calor isostérico de adsorción para los granos de kiwicha.

\begin{tabular}{ccccccccc}
\hline \multicolumn{3}{c}{ Oscar Blanco } & \multicolumn{3}{c}{ Noel Vietmeyer } & \multicolumn{3}{c}{ CICA } \\
\hline & \multicolumn{2}{c}{$\mathbf{q}_{\text {st }}$ (KJ/g) } & \multicolumn{3}{c}{$\mathbf{q}_{\mathbf{s t}}$ (KJ/g) } & \multicolumn{2}{c}{$\mathbf{q}_{\text {st }}$ (KJ/g) } \\
\hline $\mathbf{X}_{\mathbf{e}}$ (b.s.) & Expe $^{*}$ & Ajust & $\mathbf{X}_{\mathbf{e}}$ (b.s.) & Expe $^{*}$ & Ajust $^{* *}$ & $\mathbf{X}_{\mathbf{e}}$ (b.s.) & Expe $^{*}$ & Ajust $^{* *}$ \\
\hline 0,06 & 6,108 & 6,340 & 0,04 & 2,553 & 2,556 & 0,06 & 4,495 & 4,645 \\
0,08 & 3,907 & 3,047 & 0,06 & 1,950 & 1,715 & 0,08 & 2,698 & 2,051 \\
0,10 & 0,865 & 1,465 & 0,08 & 0,619 & 1,151 & 0,10 & 0,279 & 0,905 \\
0,12 & 0,371 & 0,704 & 0,10 & 0,836 & 0,772 & 0,12 & 0,227 & 0,400 \\
0,15 & 0,083 & 0,235 & 0,12 & 0,842 & 0,518 & 0,14 & 0,197 & 0,176 \\
\hline *Evaluado con la ecuación de Clausius-Clayperon ${ }^{* *}$ ecuación &
\end{tabular}

*Evaluado con la ecuación de Clausius-Clayperon, ${ }^{* *}$ ecuación de Tsami

Tabla 8. Calor isostérico de adsorción para la harina de kiwicha.

\begin{tabular}{ccrccc}
\hline \multicolumn{3}{c}{ Oscar Blanco } & \multicolumn{3}{c}{ Noel Vietmeyer } \\
\hline \multicolumn{3}{c}{$\mathbf{q}_{\mathbf{s t}}$ (KJ/g) } & \multicolumn{3}{c}{$\mathbf{q}_{\mathbf{s t}}$ (KJ/g) } \\
\hline $\mathbf{X}_{\mathbf{e}}$ (b.s.) & \multicolumn{1}{c}{ Expe $^{*}$} & \multicolumn{1}{c}{ Ajust $^{* *}$} & $\mathbf{X}_{\mathbf{e}}$ (b.s.) & Expe $^{*}$ & Ajust $^{* *}$ \\
\hline 0,05 & 31,1567 & 32,0282 & 0,06 & 26,1395 & 26,9917 \\
0,06 & 23,2364 & 21,5503 & 0,08 & 18,0327 & 15,8423 \\
0,08 & 9,3820 & 9,7565 & 0,12 & 3,3163 & 5,4575 \\
0,10 & 3,5145 & 4,4171 & 0,16 & 1,7655 & 1,8800 \\
0,12 & 1,6121 & 1,9998 & 0,20 & 0,9635 & 0,6477 \\
${ }^{*}$ Evaluado con la ecuación de Clausius-Clayperon, ${ }^{* *}$ ecuación de Tsami
\end{tabular}

En la Figura 9, se muestran los valores del calor isostérico de adsorción neto en función del contenido de humedad de equilibrio $X_{e}$, estimados de acuerdo a la ecuación de Tsami para las variedades de granos de kiwicha, se puede observar que el calor isostérico $q_{s t}$ para los granos de kiwicha Oscar Blanco disminuye de 6,340 a $0,235 \mathrm{~kJ} / \mathrm{g}$ al incrementar $X_{e}$ de 0,06 a $0,15 \mathrm{~g}$ de agua/g m.s. (Tabla 7), para Noel Vietmeyer $q_{s t}$ disminuye de 2,556 a $0,518 \mathrm{~kJ} / \mathrm{g}$ para $X_{e}$ entre 0,04 a $0,12 \mathrm{~g}$ de agua/g m.s., mientras que para la variedad $\mathrm{CICA} q_{s t}$ disminuye de 4,645 a 0,176 kJ/g, este hecho es usual en los alimentos [31], lo que indica que cuanto mayor es el contenido de agua, menor es la energía necesaria para eliminarla hasta ese nivel, ya que a valores de $X_{e}$ entre 2 a $8 \%$ el agua se encuentra fuertemente enlazada a los sitios activos de la monocapa del alimentos [45], es evidente que a menores valores de $X_{e}$ se necesita mayor energía para poder desprender moléculas de agua ligadas a zonas polares en los macronutrientes (material higroscópico) constituyentes del alimento, debido a que estos (agua) están fuertemente unidos como consecuencia de la similitud entre las fuerzas responsables de la adsorción física y las fuerzas de licuefacción (fuerzas de van der Waals), es por este motivo que es necesario una mayor energía para la liberación o remoción de estas molécula No obstante a valores menores de $10 \%$ de $X_{e}$, $q_{\text {st }}$ presenta comportamiento exponencial para las tres variedades, siendo más atenuada para la variedad Oscar Blanco, debido al contenido de fibra, por el contrario para la variedad CICA este comportamiento no es pronunciado, esto es evidente ya que mayores contenidos almidón se presentará mayores sitios activos que permitirán enlazar agua, por lo tanto se requerirá menor energía para eliminarlos. 


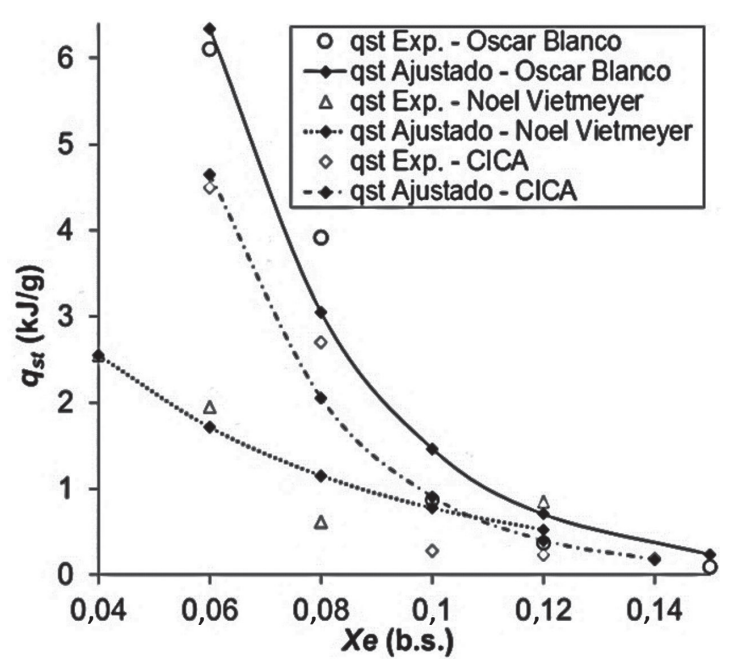

Figura 9. Calor isostérico de adsorción para los granos de kiwicha.

Tabla 9. Ecuaciones de Tsami ajustadas para la kiwicha.

\begin{tabular}{llc}
\hline Tipo/Variedad & Ecuación & $\mathbf{R}^{2}$ \\
\hline Grano - Oscar Blanco & $q_{s t}=57096,1 e^{(-X e / 0.027)}$ & 0.95 \\
Grano - Noel Vietmeyer & $q_{s t}=5678,9 e^{(-X e / 0.050)}$ & 0.84 \\
Grano - CICA & $q_{s t}=53972,3 e^{(-X e / 0.025)}$ & 0.94 \\
Harina - Oscar Blanco & $q_{s t}=232233,3 e^{(-X e / 0.025)}$ & 0.99 \\
Harina - Noel Vietmeyer & $q_{s t}=133496,2 e^{(-X e / 0.038)}$ & 0.98 \\
\hline
\end{tabular}

Para el caso de la harina de kiwicha, se observa que el $q_{s t}$ es mayor para la variedad Noel Vietmeyer (Tabla 8), y que estos son mayores en comparación al de los granos, esta diferencia se puede evidenciar en los coeficientes de las ecuaciones ajustadas para $q_{s t}$ (Tabla 9), donde $q_{0}$ es mayor para las harinas, este hecho se debe a que la harina presenta mayor superficie de contacto, exponiendo en gran parte sus componentes como ácidos grasos, proteínas, minerales, los cuales se enlazan con moléculas de agua, por lo que requieren mayor energía para su remoción, en tanto que la variedad Oscar Blanco al presentar mayor contenido de fibra permite poca migración de moléculas de agua, este hecho se evidencia en la Figura 10.

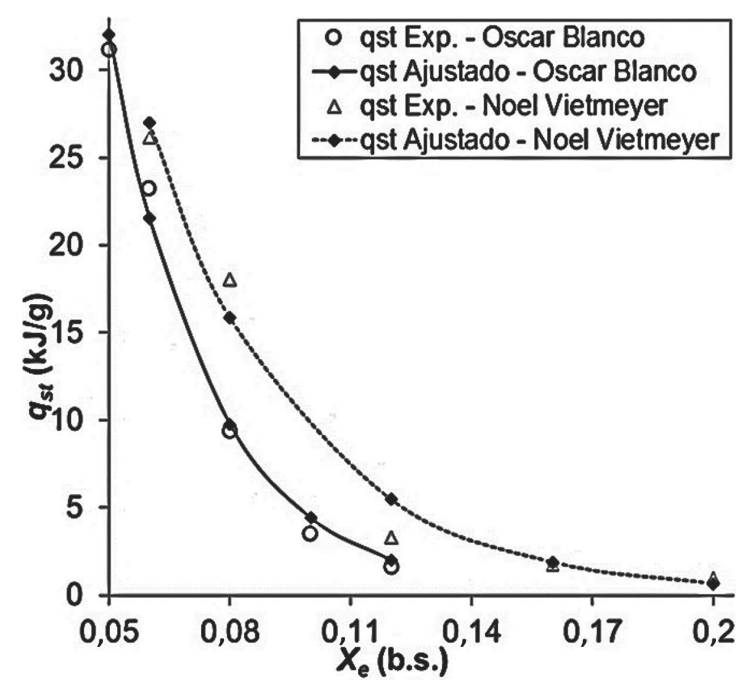

Figura 10. Calor isostérico de adsorción para la harina de kiwicha. 
Zapata et al. [47] encontró valores similares para la avena en grano (Avena sativa), PumacahuaRamos et al. [44] para almidón de quinua, así como en otros productos como bagazo de yuca (Manihot esculenta) [46], en maíz amiláceo [48] y almidón de papa [49], Heldman y Lund [18], reportaron valores del calor isostérico de sorción de $(0,078 \mathrm{~kJ} / \mathrm{g})$, para el maíz a la humedad de $0,1 \mathrm{~g}$ de agua/g m.s. y temperatura de $22^{\circ} \mathrm{C}$. Resende et al. [31], encontraron que para humedades entre 0,101 a $0,2171 \mathrm{~g}$ de agua/g m.s., $q_{s t}$, varía de 3,96 a $2,71 \mathrm{~kJ} / \mathrm{g}$, en semillas de frijol. Choque et al. [22], Encontraron valores de $q_{s t}$ que varían de 7,7022 a $0,0165 \mathrm{~kJ} / \mathrm{g}$ para el maíz morado, para una humedad de equilibrio de 0,07 a 0,17 $\mathrm{g}$ de agua/ $\mathrm{g}$ m.s., reportó que el calor isostérico disminuía con el incremento de la humedad de equilibrio, este hecho puede ser explicado considerándose que inicialmente la adsorción ocurre en el sitio disponible más activo, dando origen a una alta energía de interacción [29]. El aumento del calor isostérico al disminuir la humedad puede explicarse teniendo en cuenta que inicialmente la adsorción tiene lugar en los sitios disponibles más activos, lo cual lleva aparejado elevadas energías de sorción.

\section{Conclusiones}

Las isotermas de adsorción permitieron evaluar las condiciones de almacenamiento del grano y harina de kiwicha de las variedades Oscar Blanco, Noel Vietmeyer y CICA, para intervalos de humedad relativa de 10 a $90 \%$ a las temperaturas de $18^{\circ} \mathrm{C}$, $20^{\circ} \mathrm{C}, 25^{\circ} \mathrm{C}$ y $30^{\circ} \mathrm{C}$.

La temperatura tiene efecto sobre humedad de equilibrio $\left(X_{e}\right)$ para las dos variedades de kiwicha, cualquier cambio de temperatura influenciara significativamente en la humedad de equilibrio y esta última está relacionada directamente con la actividad del agua a través de las isotermas de adsorción.

Se han estudiado 11 modelos matemáticos, de ellos el modelo de Adam y Shove se ajusta de mejor manera a las isotermas de adsorción para ambas variedades de kiwicha a las temperaturas de $18^{\circ} \mathrm{C}$, $20^{\circ} \mathrm{C}, 25^{\circ} \mathrm{C}$ y $30^{\circ} \mathrm{C}$. Los parámetros fisicoquímicos del proceso de adsorción se calcularon a partir del modelo de $G A B$, y permitieron evaluar las constantes $C_{G A B}$, ky $X_{m}$ para los granos de kiwicha. El calor isostérico de adsorción permitió conocer la energía necesaria para alcanzar un determinado contenido en humedad durante el proceso de adsorción en los granos y harina de kiwicha, siendo mayor para esta ultima.

\section{Agradecimiento}

A la Universidad Nacional José María Arguedas de Andahuaylas que a través de la Vicepresidencia de Investigación, que apoyo con el financiamiento parcial para el desarrollo de la investigación.

\section{Referencias bibliográficas}

[1] Higinio R. Elaboración de una mezcla instantánea de arroz (Oryza sativa), cañihua (Chenopodium pallidicaule Aellen) y kiwicha (Amaranthus caudatus) por el método de cocción extrusión. (Tesis de grado) Lima, Perú: UNAC; 2011.

[2] Chipana M, Stuva C. Kiwicha producto de exportación en el Perú. Articulo ministerio de la producción, Perú; 2005.

[3] Huamán N. Predicción de la actividad de agua utilizando el modelo matemático de G.A.B. en el proceso de deshidratación osmótica del capulí (Phisalis peruviana) (Tesis de maestría) Lima, Perú: UNALM; 2001.

[4] Moreira R, Chenlo F, Torres MD, Vallejo N. Thermodynamic analysis of experimental sorption isotherms of loquat and quince fruits. J Food Eng. 2008;88:514-21.

[5] Brennan JG. Manual del procesado de los alimentos. España: Edit. ACRIBIA; 2008.

[6] Orrego AC. Procesamiento de alimentos,1era edición. Colombia: Universidad Nacional de Colombia - Manizales; 2003.

[7] Li Y, Wang X, Jiang P, Li X. Sorption equilibrium moisture and isosteric heat of adsorption of chinese dried wheat noodles. Journal of Stored Products Research. 2016; 67:19-27.

[8] Raji AO, Ojediran JO. Moisture sorption isotherms of two varieties of millet. Food Bioprod Process. 2011;89(3):178-84.

[9] Fellows P. Food Processing Technology Principles and Practice. 2da edición. Edit. TJ International. England; 2000.

[10] Blahovec J, Yanniotis S. Modified classification of sorption isotherms. J Food Eng. 2009;91(1):72-77.

[11] Yan Z, Sousa-Gallagher M, Oliveira F. Sorption isotherms and moisture sorption hysteresis of intermediate moisture content banana. J Food Eng. 2008;86(3):342-48.

[12] Tolaba MP, Peltzer M, Enríquez N, Pollio ML. 
Grain sorption equilibria of quinoa grains. J Food Eng. 2004;61(3):365-71.

[13] Lamharrar A, Idlimam A, Ethmare Kane CS, Jamali A, Abdenouri N, Kouhila M. Sorption isotherms and drying characteristics of Artemisia arborescens Leaves. Journal of Agronomy. 2007;6:488-98.

[14] Tsami E, Maroulis Z, Marinos KD, Saravacos G. heat of sorption of water in dried fruits. International Journal of Food Science and technology. 1990;25(3):141-5.

[15] Labuza TP, Kaanane A, Chen J. Effect of temperature on the moisture sorption isotherm and water activity shift of two dehydrated food. J. Food Sci. 1985;50(2):385-91.

[16] Soleimani M, Tabil L, Shahedi M, Emani S. Sorption isotherm of hibrid seed corn. The canadian society for engineering in agricultural, food, environmental, and biological systems CSBE. 2006.

[17] Toloaba, M. P., Peltzer, M., Enriquez, N., Pollio, M. L. Grain sorption equilibrium of quinoa grains. J. Food Eng. 2004; 61(3):365-371.

[18] Heldman, D., Lund D. Handbook of food engineering. 2da edic. Editorial. CRC Press. Florida - EE. UU.; 2007.

[19] Shafiur, R. Manual de conservación de los alimentos. Editorial ACRIBIA - España; 2003.

[20]Viades, T. Adsorción de agua en alimentos. Isoterma de adsorción de Guggenheim, Anderson y de Boer (GAB). Seminario de Investigación: Fenómenos de superficie, Postgrado en Ciencias Químicas, UNAM México; 2008.

[21] Chasquibol, S., Delmas, R., Rivera, C., Lengua, C., Aguirre, M. Bazán, G., Becerra, V., et al. Contribución a la normalización de productos tradicionales andinos: Maca, Kiwicha, Cañihua, Mashua. (Tesis de grado), Lima, Perú: UNMSM; 1999.

[22] Choque D.; Llalla C.; Solano A.M.; Ligarda C.A. Modelamiento matemático de las isotermas de adsorción de la quinua (Chenopodium quinoa) variedad blanco de Junín (Tesis de grado), Cusco, Perú: UNSAAC; 2010.

[23] Choque D. Evaluación de las isotermas de adsorción del maíz morado. (Tesis de maestría), Cusco, Perú: UNSAAC; 2009.

[24]Brett, B., Figueroa, M., Sandoval, A.J., Barreiro, J.A., Müller, A.J. moisture Sorption characteristics of starchy products: oat flour and rice flour. Food Biophys. 2009; 4(3):151157.
[25] Alvarado, J.; Aguilera, J.M. Métodos para medir las propiedades físicas en la industria de los alimentos, Editorial Acribia, S.A.; 2001.

[26]Bell, L. N., Labuza, T. P. Moisture sorption. Practical aspects of isotherm measurement and use. 2nd Edition. Ed. AACC; 2000.

[27] García, L.; Kobylansky, J.; Pilosof, A. Modelling water sorption in okara soy milk. Drying Technology. 2000; 18(9): 2091-2103.

[28] Prieto, F., Gordillo, A., Prieto, J., Gómez, C., Román, A. Evaluación de las isotermas de sorción en cereales para desayuno. Sociedad Mexicana de Ciencia y Tecnología de Superficies y Materiales; 2006.

[29]Zug, J. Fisicoquímica Especial. Isoterma de sorción de tres etapas y modelos de sorción restringida. Fac. Ing. UBA. Argentina; 2002.

[30] Chen, C. Obtaining the isosteric sorption heat directly by sorption isotherm equations. J. Food Eng. 2006; 74(2):178-185.

[31]Resende, O., Correa, C. P., Gonell, L. D., Ribeiro, M. D. Isotermas e Calor Isostérico de sorção do feijão. Food Science and Technology. 2006; 26(3):626-631.

[32] Gabas, A.L. Secagem de uva Itália em leito fixo. (Tese de mestrado); São Paulo, Brasil: Faculdade de Engenharia de AlimentosUNICAMP; 1998.

[33] Van Den Berg, C., Bruin, S. Water activity and its estimation in food systemas. In L.B. Rockland. F., Steward (Eds.). Water Activity: Influence on food quality. Academic Press, New York; 1981.

[34] Kaya, S., Kahyaoglu, T. Thermodynamic properties and sorption equilibrium of pestil (grape leather). J Food Eng. 2005; 71: 200207.

[35] Raji AO, Ojediran JO. Isotermas de sorción de humedad de dos variedades de mijo. Proceso de bioprod de alimentos. 2011; 89(3):178-184.

[36] Blahovec J, Yanniotis S. Clasificación modificada de las isotermas de sorción. J Food Eng. 2009; 91(1):72-77.

[37] Oswin CR. La cinética de la vida del paquete III. Isoterma. J Soc Chem Ind. 1946; 65(12):419421.

[38] Sopade, P. A., Ajisegiri, E. S. Moisture sorption study on Nigerian foods: maize and sorghum. Journal of Food Process Engineering. 1994; 17(1):33-56.

[39]Galvez, A., Aravena, E., Mondaca, R. Isotermas de adsorción en harina de maíz (Zea mays L.). Food Science and Technology. 
2006; 26(4):821-827.

[40] Chirife, J., Iglesias, H. A. Equations for fitting water sorption isotherm of foods: Part I: a review. Journal of Food Technology. 1978; 13:159-174.

[41]Andrade, R.R., Lemus, R.M., Pérez, C.E. Models of sorption isotherms for food: uses and limitations. Vitae. 2011; 18(3):325-334.

[42] Chung, D.S., Lee, CH. Adsorption and desorption of water vapor by cereal grain and their products. Part II: Development of the general isotherm equation. Transaction of the ASAE. 1985; 10:552-555.

[43] Torrealba, M.A. Determination of green/unripe plantain pre-cooked flour humidity sortion isoyherms (Musa paradisiaca L.). Revista AGROLLANIA. 2007; 4:9-22

[44] Pumacahua-Ramos, A., Limaylla-Guerrero, K.M., Telis-Romero, J., Lopes J.F. Water adsorption isotherms and isosteric heat of quinoa starch. Biotecnología en el sector agropecuario y agroindustrial. $2017 ; 15(1)$ :95104.

[45] Tsami, E. Heat of sorption of water in dried fruits. International Journal of Food Science and Technology. 1991 25(3):350-359

[46] Polachini, T.C., Betiol, L.F.L., Lopes-Filho, J.F., Telis-Romero, J. Water adsorption isotherms and thermodynamic properties of cassava bagasse. Thermochimica Acta. 2016; 632:79-85.

[47]Zapata, J.E., Quintero, O.A., Porras, L.D. Isotermas de sorción para avena (Avena sativa) en grano. Rev. Agron. 2015; 23(1):8292.

[48] Samapundo, S., Devliegher, F., De MeuleNAer, B., Atakwase, A., Lamboni, Y., Debevere, J.M. Sortion isotherms and isosteric heats of sorption of whole yellow dent corn. J Food Eng. 2007; 76:168-175.

[49] Al-Muhtaseb, A.H. McMinn, W., Magge, T.R.A. Water sorption isotherm of starch powders Part 1: Mathematical description of experimental data. J Food Eng. 2004; 61:297-307.

[50] Arthur, E., Tuller, M., Moldrup, P., De Jongel L.W. Evaluation of theoretical and empirical water vapor sorption isotherm models for soils, Water Resour. Res. 2016; 52:190-205. 